\title{
Do melancholic and atypical specifiers reduce heterogeneity in major depressive disorder?: A secondary analysis of the $\mathrm{STAR}^{*} \mathrm{D}$ trial
}

Lorenzo Lorenzo-Luaces, $\mathrm{PhD}^{1}$, John F. Buss, $\mathrm{BA}^{1}$, Eiko I. Fried, $\mathrm{PhD}^{2}$,

1 Department of Psychological and Brain Sciences, Indiana University, Bloomington, IN, USA

2 Department of Psychology, Leiden University, Leiden 2333 AK, Netherlands

Correspondence regarding this article should be directed to Lorenzo Lorenzo-Luaces, lolorenz@iu.edu 


\section{Highlights}

- Paradoxically, specifiers for depression may increase heterogeneity

- In $\mathrm{STAR}^{*} \mathrm{D}$ data, melancholia does not reduce heterogeneity

- Atypical appears to reduce heterogeneity, but only because it is less frequently endorsed

\section{Considerations}

- We did not include patients with depression but who were not seeking treatment nor patient with psychotic, schizoaffective or bipolar depression

- We did not explore the validity of the categories 


\begin{abstract}
Objectives: Specifiers for the diagnosis of a major depressive disorder (MDD), like melancholic and atypical features, are supposed to reduce heterogeneity in symptom presentation. However, Fried et al. (2020) recently quantified how, at least the melancholic specifier, could increase heterogeneity in presenting symptoms. Methods: We analyzed baseline data from the Inventory of Depression Symptoms in the Sequenced Alternatives to Relieve Depression (STAR*D) trial. IDS data were available for 3, 724 patients, of which 2, 498 reported five symptoms of depression on the IDS ("IDS-MDE"). For patients with IDS-MDE, we differentiated between those with melancholic, non-melancholic, atypical, and non-atypical depression as per the IDS. For each of these groups, we calculated the number of unique combinations of the symptom that allowed a patient to meet criteria for their given diagnostic group, as well as the number of unique combination of depressive symptoms (i.e., ignoring the melancholic and atypical symptoms). Results: There was more heterogeneity within the specifiers than in the full sample. When considering only the symptoms of depression, there was somewhat less heterogeneity in the melancholic than non-melancholic groups. However, most symptom profiles, even in the specifier subgroups, had five or fewer individuals, suggesting the presence of significant heterogeneity.
\end{abstract}

Conclusion: The DSM specifiers largely appear to reduce heterogeneity by creating smaller subgroups of patients with infrequent symptoms but there is a large degree of heterogeneity present even among those who meet the specifier criteria. Moreover, the specifier criteria themselves may add heterogeneity. 


\section{Introduction}

Experiences of depressed mood or low positive affect can range from states of transient sadness to highly debilitating, chronic, and recurrent patterns of symptoms (Lorenzo-Luaces, 2015; Lorenzo-Luaces et al., 2017). According to the Diagnostic and Statistical Manual of Mental Disorders (DSM), a major depressive episode (MDE) requires a minimum of five symptoms, one of which must be depressed mood or anhedonia (i.e., loss of interest or pleasure) for at least two weeks (American Psychiatric Association, 2013). Most MDE symptoms are compound criteria that vary qualitatively (e.g., diminished ability to think, or to concentrate, and indecisiveness are all counted as the same symptom") or consist of complaints in the opposite direction (e.g., insomnia or hypersomnia). An MDE is frequently categorized as major depressive disorder (MDD), the most commonly diagnosed depressive disorder (Zimmerman et al., 2006), if criteria are not met for bipolar I or II, schizoaffective disorders, and it is deemed that general medical conditions and substances do not account for the symptoms.

The optimal classification of MDD has been one of the major challenges in the history of psychiatry (Fried \& Nesse, 2015b; Lorenzo-Luaces, 2015; McGlinchey et al., 2006; Parker, 2005), despite being one of its most widely researched questions (Cai et al., 2020; Horwitz \& Wakefield, 2007; Shorter, 2007). Questions include both the optimal differentiation of MDD from "normal" depressed mood (Wakefield \& Schmitz, 2013a, 2013b, 2013c; Wakefield et al., 2007), as well as identification of the various pathological states, sometimes conceptualized as specifiers or endophenotypes, likely subsumed under the diagnosis of MDD (Shorter, 2007). Because the DSM diagnostic criteria for a MDE, and thus MDD, list a polythetic set (i.e. there are more symptoms than necessary for a diagnosis), there can be considerable heterogeneity in the symptomatic presentation of two different patients who meet criteria for the diagnosis. Two individuals may have a diagnosis of MDD diagnoses and not overlap any one symptom (Fried \& Nesse, 2015a; Zimmerman et al., 2015). Being very conservative (i.e., counting compound criteria as a single symptom), there are 227 unique ways of meeting 
criteria for a MDE, of which patients appear to meet most combinations (Fried \& Nesse, 2015a; Zimmerman et al., 2015). Being more liberal, and perhaps more accurate, in counting the most qualitatively different compound symptoms as distinct symptoms, there are as many as 10,377 ways of meeting MDE criteria (Fried et al., 2020).

The DSM recognizes that its polythetic diagnoses lead to heterogeneity in the symptom presentations of patients meeting criteria for the same diagnosis. Accordingly, the manual provides the option to identify "more homogeneous" subgroups via subtypes and specifiers. Subtypes are defined as mutually exclusive categories like "predominantly hyperactive/impulsive" attention-deficit and hyperactivity disorder (ADHD) vs. "predominantly inattentive" ADHD. Specifiers are not mutually exclusive, such as seasonal-affective and atypical depression. For the diagnosis of a MDE, the DSM differentiates between diagnostic categories (i.e., bipolar vs. unipolar depressive disorders), illness history (i.e., recurrent vs. single episode), and symptom severity (i.e., mild, moderate, and severe) while containing nine different specifiers for course or symptom presentation (e.g., catatonia, anxious distress). Of these specifiers, melancholia and atypical depression features are among the oldest and most widely studied (Kessing, 2007), and are the focus of the present paper. Below we test the assumption that they result in more homogeneous groups of patients than MDD, i.e. if they are working as intended. Consistent with the DSM (APA, 2013), we refer to melancholia and atypical depression as specifiers, not subtypes as they are sometimes referred to.

Melancholia is characterized primarily by a loss of positive affectivity manifested either in the loss of pleasure in almost all activities or a lack of mood improvement in the context of positive events. The melancholic specifier was first formally operationalized in DSM-III (Association et al., 1980). The specifier was meant to capture a long-standing distinction in the history of conceptualizing depression, which differentiated between milder forms of depression, usually assumed to be psychogenic or triggered by a negative event, and depression without a cause (Horwitz \& Wakefield, 2007; Horwitz et al., 2016; Shorter, 2007). Melancholia is commonly cited in the literature as being a sub-classification of depression whose onset 
and maintenance has a greater contribution from biological vulnerabilities (Parker, 2005).

Atypical depression, on the other hand, differs from the more endogenous melancholia and is characterized primarily by endorsing less common directions of MDE symptoms, such as increased (rather than decreased) appetite or weight, or hypersomnia (rather than insomnia). The "atypical features" specifier was formally introduced in DSM-IV (Association et al., 2000), but comports to prior publications which identified a subgroup of patients who may have a differential response to treatment (Pae et al., 2009), though this pattern of results has not been replicated (Cuijpers et al., 2017; Uher et al., 2011). The DSM imposes a hierarchical rule upon the atypical specifier wherein an individual cannot be diagnosed with atypical depression if they meet criteria for the melancholic specifiers. More detailed information on the history and validity of melancholia and atypical depression can be found elsewhere (Baumeister \& Parker, 2012; Stewart et al., 2007; Türkçapar et al., 1999).

After decades of contentious discussions regarding the validity of melancholia and atypical depression, many questions remain unresolved. These include whether they are distinct when hierarchical criteria are relaxed (Stewart et al., 2007), whether they consistently predict treatment outcomes (Bühler et al., 2014; Cuijpers et al., 2017; Łojko \& Rybakowski, 2017), and whether they are (differentially) predicted by biomarkers (Juruena et al., 2018). While the predictive validity of the specifiers may be in question, it is still possible that, as the DSM states, they identify more homogeneous subgroups of patients. If this is the case, the specifiers may still one day validly predict important clinical variables, such as undiscovered biomarkers, or responses to newly developed treatments. A recent challenge to this assumption was made by Fried et al. (2020) who computed the total number of possible symptom profiles for MDD vs MDD plus the melancholic specifier. The number of possible symptom profiles for meeting a MDE criteria ranged from 227 to 10,377, depending on how "compound" symptoms were treated (e.g., whether psychomotor agitation and retardation were conceptualized as two different symptoms or just one manifestation of motor disturbances). However, the total number of symptom profiles for MDD plus melancholia ranged 
from 10,999 to 341,737 .

These calculations demonstrate that there are more potential ways to meet for a MDE plus melancholia than a MDE alone, which contrasts with the DSM's explicit goal of using melancholia as a specifier to identify a more homogeneous group of patients. Importantly, it is unclear whether, in practice, patients exhibit more symptom combinations of MDD plus melancholia vs. MDD alone. While we are not aware of work being done on unique symptom profiles in melancholia, there is prior work for MDD. Zimmerman et al. (2015) described how, out of a total of 227 possible symptom profiles, if one was counting compound symptoms as a single symptom (e.g., hypersomnia and insomnia count as the same symptom), 170 were represented from a sample of 1,566 patients (74.9\%). Fried \& Nesse (2015) investigated unique symptom profiles in a sample of 3,703 patients with MDD, and went beyond the 9 DSM symptoms in that they separated insomnia vs hypersomnia, psychomotor agitation vs retardation, and weight vs appetite changes. Of 4,096 possible combinations, 1,030 were identified in the data $(25.1 \%)$.

To our knowledge, no empirical research has quantified whether the MDE specifiers melancholic and atypical depression reduce heterogeneity. We sought to explore this question, using data from the Sequenced Treatment Alternatives to Relieve Depression (STAR*D) Study (Rush et al., 2004), in which patients were administered the 30-item Inventory of Depressive Symptomatology (IDS), which includes questions of the DSM-MDE symptoms, as well as the melancholic and atypical specifiers. We followed the procedures used in prior work (Fried \& Nesse, 2015a; Zimmerman et al., 2015), which involve counting the number of unique combinations of symptoms endorsed by patients. We refer to this unique combination of symptoms as "profiles."

\section{Methods}

\section{$\mathrm{STAR}^{*} \mathrm{D}$}

We reanalyzed the public-access dataset from the NIH-supported Sequenced Treatment 
Alternatives to Relieve Depression (STAR*D) study (Rush et al., 2004), which we downloaded from the National Institute of Mental Health Data Archive on September 16, 2019. $\mathrm{STAR}^{*} \mathrm{D}$ was a multi-site clinical trial conducted in the USA and designed to have greater external validity than treatment trials usually do. STAR*D treatment was designed as a stepped care protocol wherein patients received additional, usually more intensive, treatments if their symptoms had not improved at a prior level. In the first level stage, 4,041 patients were enrolled, and all participants received the selective serotonin reuptake inhibitor (SSRI) citalopram. Data were collected via telephone interviews. STAR*D was approved by the institutional review boards (IRBs) of all participating institutions, and after complete description of the study to the subjects, written informed consent was obtained. Prior $\mathrm{STAR}^{*} \mathrm{D}$ publications report on a subset of the 4,041 patients; excluding those who had mild symptoms or who did not provide data beyond the initial assessment (Trivedi et al., 2006). Because our research question does not involve change in symptoms, and because we want to maximize the representativeness of our sample (i.e., to include those with mild symptoms) we did not exclude any patients from our analysis a priori.

\section{Participants}

Inclusion criteria for $\mathrm{STAR}^{*} \mathrm{D}$ participants were: being between the ages of 18 and 75 years, meeting DSM-IV criteria for unipolar, non-psychotic MDD. MDD status was assessed by a checklist based on DSM-IV criteria (Fava et al., 2003; Rush et al., 2004), after patients expressed interest in treatment for depression. Exclusion criteria were a history of mania or hypomania, schizophrenia, schizoaffective disorder, or psychosis, or current anorexia, bulimia, or primary obsessive-compulsive disorder (OCD), which were assessed with The Psychiatric Diagnostic Screening Questionnaire (Zimmerman \& Mattia, 2001) via clinical interview. Further exclusion criteria and details about the study design are described elsewhere (Fava et al., 2003; Rush et al., 2004). Patients in STAR*D were excluded from some publications if they had scores $\leq 14$ on the Hamilton Rating Scale for Depression (HRSD) (Trivedi et al., 2006), though these patients were assessed for baseline and their data was 
available at subsequent steps (Rush et al., 2004). We analyze data for all STAR*D participants who had available IDS scores, even if they entered the trial with milder symptoms.

\section{Outcome measures}

Inventory of Depressive Symptomatology (IDS). We analyzed baseline data on the clinician-rated version of the IDS (Rush et al., 1996). The IDS-C encompasses 30 depression symptoms, both DSM and non-DSM symptoms, rated on a 4-point (0-3) scale with a higher score indicating greater severity. Consistent with prior work (Ulbricht et al., 2018), we considered a symptom to be present when an individual endorsed a severity level $\geq 2$. The IDS covers most DSM-5 criterion symptoms in disaggregated form. For example, it queries both psychomotor agitation and psychomotor retardation. Nonetheless, we had to make several decisions regarding which variables to include in our analyses, discussed below.

Appetite or weight disturbances. Disaggregated information were not available for the two symptom domains "weight problems" and "appetite problems." Instead, a patient was deemed to have either increases or decreases appetite or weight but could not rate both increases and decreases. For analyses focused on the We combined the responses to the appetite and weight questions, using the highest rating on either question to create two variables: appetite/weight decrease or appetite/weight increase. For example, if a participant was judged to have experienced weight loss, that individual was rating as having appetite/weight decrease but not appetite/weight increase. We code the variables this way to avoid adding unnecessary heterogeneity (e.g., so two individuals who had severe appetite loss were not deemed as having a different symptom profile if one participant lost weight but the other did not).

Sleep disturbances. The IDS queries early, middle, and late insomnia as well as hypersomnia. We generally distinguished hypersomnia from insomnia. Distinguishing early insomnia from middle and late insomnia is necessary for the diagnosis of melancholic features. Nonetheless, to avoid inflating the degree of heterogeneity present in the symptom data, we consider all these examples of insomnia as a single symptom, as done in prior work 
(Fried \& Nesse, 2015a), assigning each patient the higher-rated symptom they endorsed (i.e., if the highest symptom was $\geq 2$ the patient was considered to have insomnia). We separately explored the presence of the early insomnia vs. other symptoms of insomnia only when counting the number of symptom profiles from melancholic and depressive symptoms.

\section{Analytic strategy}

All data were analyzed using the $\mathrm{R}$ programming language (code available at: https:// osf.io/v8sbe/). From the 4,041 participants originally enrolled into STAR*D, 3,867 (95.69\%) patients provided data during the first measurement point of the first treatment stage. We had full symptom-level data for the IDS on 3,724 patients, who represented $92.16 \%$ of all patients. As the sample size was large, we performed listwise deletion for patients with missing IDS data. Our aim was to count the number of symptom profiles across MDD and its melancholic and atypical specifiers.

First, we present basic descriptive data on the categorical endorsement of all the symptoms we are studying, which include the symptoms from the DSM MDE criteria, the symptoms from the melancholic specifier, and the symptoms from the atypical specifier. We identify a subsample of patients who met criteria for MDD $(2,498,61.82 \%)$ using the IDS. This is lower than the number of cases with MDD in other $\mathrm{STAR}^{*} \mathrm{D}$ reports because we rely on the IDS rather than the STAR*D-specific checklist Rush et al., 2004.

To emulate the ways in which the DSM uses the specifiers, we created five groups (See Figure 1). The IDS-MDE group consisted of patients who endorsed either sadness, loss of interest, or loss of pleasure, and a total of five DSM MDE symptoms. The second group, IDSmelancholic, featured patient who met IDS-MDE criteria plus who endorsed the melancholic specifier criteria. The melancholic criteria require the presence of either loss of pleasure or loss of mood reactivity, along with three symptoms from a list that includes: distinct quality of mood, depression that is worse in the morning, early-morning awakenings, psychomotor agitation or retardation, anorexia or weight loss, and excessive or inappropriate guilt. The third group was of patients who met IDS-MDE criteria but did not meet the melancholic 
specifier criteria ("IDS non-melancholic"). The fourth group was of patients who met IDSMDE criteria and met criteria for the atypical specifier. The atypical specifier requires the presence of mood reactivity along with two other symptoms from a list of four: weight gain or increase in appetite, hypersomnia, heavy or leaden feelings in the extremities, and a long pattern of interpersonal rejection sensitivity outside the context of mood episodes. for our IDS-atypical group. We respected the DSM's hierarchical rules wherein a person cannot meet criteria for the atypical specifier if they meet criteria for the melancholic specifier. The final group was patients with non-melancholic, non-atypical depression ("IDS non-atypical").

Figure 1: Subgroups of patients derived from the IDS on $\mathrm{STAR}^{*} \mathrm{D}$

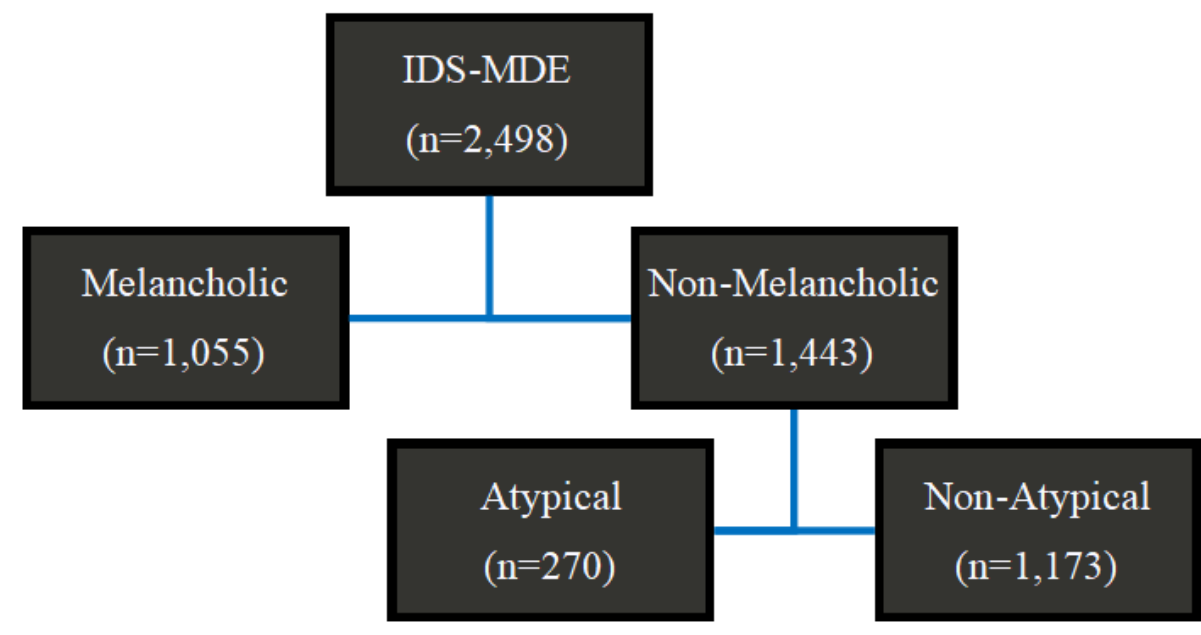

For these 5 groups, we counted the total number of symptom combinations that created the groups, using the distinct command in the dplyr package. We counted the number of symptoms relevant to each diagnosis. For the IDS-MDE group, we counted the total number of combinations of depressive symptom profiles using the DSM criteria for depression. For the IDS-melancholic and IDS non-melancholic groups, we counted the total number of combinations of melancholic and depressive symptoms. For the IDS-atypical and IDS nonatypical groups, we counted the total number of combinations using atypical and depressive symptoms.

In addition to counting the number of symptom profiles of MDD symptoms plus the 
specifier symptoms, we counted the total number of profiles of only the MDE symptoms (e.g., sadness, adhedonia, difficulty concentrating, etc). This was done for the IDS-MDE, IDS-melancholic, IDS non-melancholic, IDS-atypical, and IDS non-atypical groups. These analyses are to ensure that any differences between the groups is not due to differences in the number of symptoms considered. Additionally, we conducted several sensitivity analyses. First, we count the number of symptom profiles that are reported by 5 or more individuals in each of the five subgroups. Finally, to equate the five groups we are comparing on sample size, we randomly subsampled 100 patients from each of the five subgroups 1,000 times. This procedure provides a mean number of profiles per 100 patients for each of the diagnostic subgroups, along with a distribution of the mean number of profiles.

\section{Results}

Full IDS data were available for 3,724 patients, representing $92.16 \%$ of the full sample. Table 1 shows the descriptive statistics representing endorsement of IDS symptoms as binary with the presence $(\geq 2)$ or absence $(0-1)$ of symptoms. Roughly two third of patients (67.08\%) who had full IDS item-level data met MDD criteria on the IDS ( $\mathrm{n}=2,498$; i.e., sad mood or anhedonia and a total of five symptoms of depression). We focus our analyses on these patients. As seen in Table 1, sad mood and (93.40\%) and insomnia (91.11\%) were the most frequently reported symptoms. The least frequently reported symptoms were psychomotor retardation (9.24\%) and hypersomnia (14.81\%). Of the patients with IDSMDE, 1055 met criteria for melancholia (42.57\%), and 270 met criteria for the atypical specifier (10.90\%).

\section{Heterogeneity added by specifier symptoms}

The 2,498 patients with an IDS-MDE met the symptom criteria a total of 665 ways, for a ratio of 3.76 patients per one profile. The most common $(n=119)$ symptom profile featured the following 8 symptoms: sad mood, loss of interest, loss of pleasure, appetite/weight 
Table 1: Endorsement of specific symptoms of DSM criteria for major depression, melancholia, and atypical specifiers in patients with MDD, MDD with melancholia features, and MDD with atypical features, as determined by the IDS

\begin{tabular}{|c|c|c|c|c|c|c|}
\hline Symptom & $\begin{array}{c}\text { IDS-MDE } \\
\%\end{array}$ & $(\mathbf{n})$ & $\begin{array}{c}\text { IDS-Mel } \\
\%\end{array}$ & $(\mathbf{n})$ & $\begin{array}{c}\text { IDS-Aty } \\
\%\end{array}$ & $(\mathbf{n})$ \\
\hline Sad mood & 93.39 & 2333 & 94.12 & 993 & 90.00 & 243 \\
\hline Lost interest & 79.38 & 1983 & 82.27 & 868 & 70.00 & 189 \\
\hline Lost pleasure ${ }^{a}$ & 60.37 & 1508 & 79.72 & 841 & 35.56 & 96 \\
\hline Apettite/weight decrease ${ }^{a}$ & 45.20 & 1129 & 64.27 & 678 & 21.11 & 57 \\
\hline Apettite/weight increase ${ }^{b}$ & 27.22 & 680 & 19.62 & 207 & 57.78 & 156 \\
\hline Insomnia & 91.11 & 2276 & 95.45 & 1007 & 86.67 & 234 \\
\hline Hypersomnia $^{\mathrm{b}}$ & 14.81 & 370 & 11.47 & 121 & 32.96 & 89 \\
\hline Psychomotor retardation $^{\mathrm{a}}$ & 9.25 & 231 & 14.79 & 156 & 6.67 & 18 \\
\hline Psychomotor agitation $^{a}$ & 30.42 & 760 & 47.11 & 497 & 20.37 & 55 \\
\hline Fatigue & 80.50 & 2011 & 79.72 & 841 & 82.96 & 224 \\
\hline Worthlessness/guilt ${ }^{\mathrm{a}}$ & 76.62 & 1914 & 86.73 & 915 & 75.56 & 204 \\
\hline Diminished concentration & 87.99 & 2198 & 88.72 & 936 & 87.04 & 235 \\
\hline Suicidality & 19.82 & 495 & 25.59 & 270 & 12.59 & 34 \\
\hline Unreactive mood & 57.53 & 1437 & 76.68 & 809 & 0.00 & 0 \\
\hline Distinct quality of mood & 49.72 & 1242 & 64.83 & 684 & 47.78 & 129 \\
\hline Depression worse AM & 10.85 & 271 & 17.25 & 182 & 6.30 & 17 \\
\hline Early morning awakening & 49.84 & 1245 & 72.32 & 763 & 34.07 & 92 \\
\hline Mood reactivity & 42.47 & 1061 & 23.32 & 246 & 100.00 & 270 \\
\hline Leaden paralysis & 32.27 & 806 & 33.18 & 350 & 59.26 & 160 \\
\hline Rejection sensitivity & 51.84 & 1295 & 57.35 & 605 & 78.52 & 212 \\
\hline
\end{tabular}

decrease, insomnia, fatigue, feelings of worthlessness/guilt, and diminished concentration (i.e., all symptoms except suicidal. The next-most common symptom profile $(n=84)$ was identical but lacking in changes in appetite/weight. Most of the MDD symptom profiles (85.56\%) were endorsed by five or fewer patients.

1,055 patients who met the IDS-melancholic criteria presented with a total of 646 unique symptom combinations of depressive and melancholic symptoms (see Fig. 2), for a ratio of 1.63 patients per one profile. So, although patients who met for IDS-melancholia reported fewer depressive and melancholic symptom profiles on the IDS (646) than patients with IDS non-melancholic depression (891), melancholia did not substantially decrease heterogeneity relative to the diagnosis of MDD, though the IDS-melancholic sample was much smaller. Most of the IDS-melancholic profiles (96.59\%) were endorsed by five or fewer patients.

Patients who met IDS-atypical $(n=270)$, only reported 198 profiles, for a ratio of 1.36 patients per one profile. As before, most of the symptom profiles (98.48\%) were only endorsed by five or fewer patients. 
Figure 2: Number of unique profiles of depressive symptoms, as well as symptoms relevant to each specifier, for patients with MDD, with or without melancholic features, and with or without atypical features

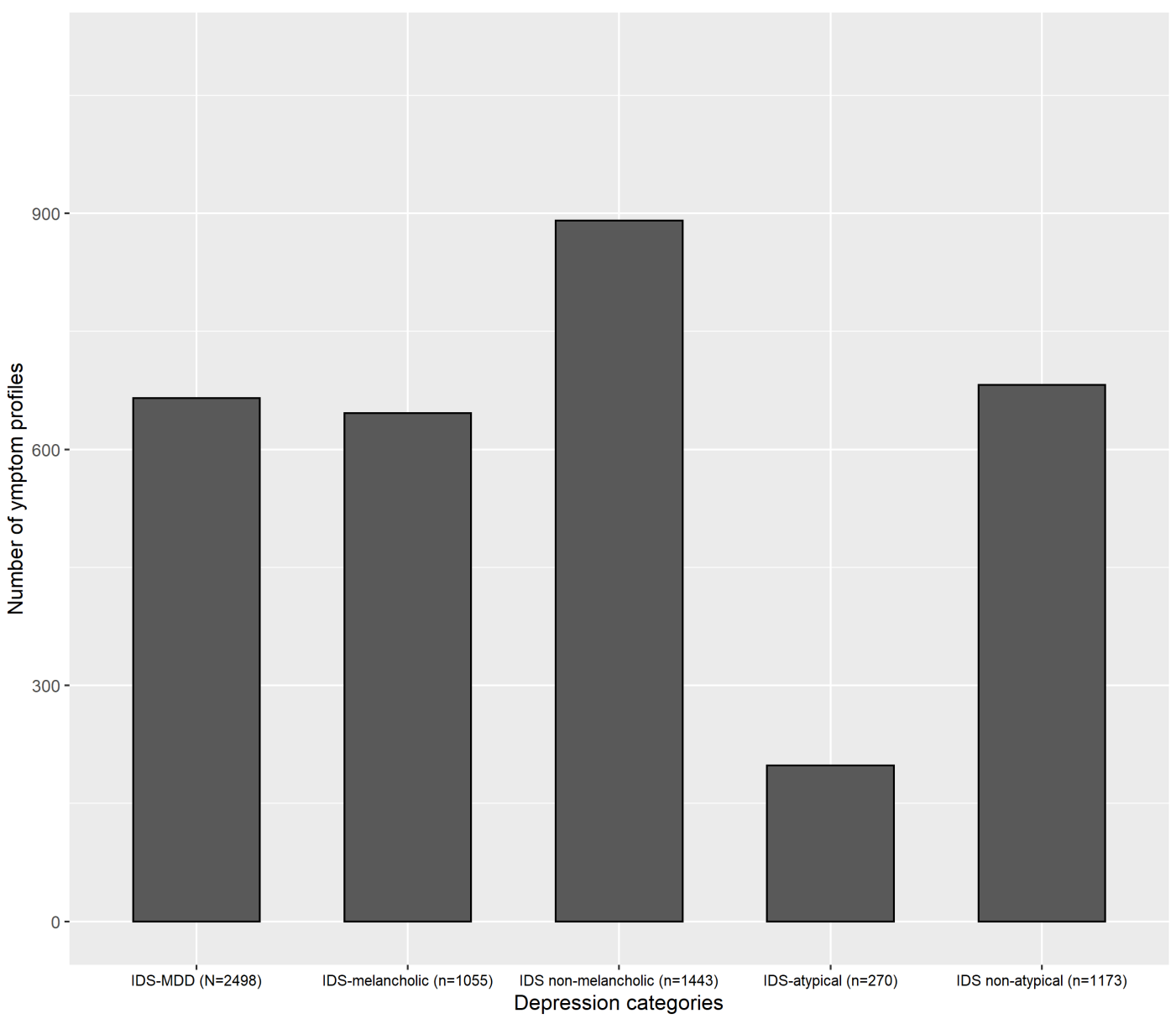

\section{Heterogeneity in depression symptoms alone}

In the previous analyses, we investigated how specifiers could influence the number of unique symptom profiles reported by patients, though the specifiers differ in the the number of symptoms they include and may thus be associated with different levels of heterogeneity as quantified by symptom count. To address this, we computed the total number of combinations of the nine symptoms of a MDE in the IDS-MDE group, IDS-melancholic, IDS-non-melancholic, IDS-atypical, and IDS non-atypical (see Fig. 3). IDS-melancholia patients had fewer unique combinations of MDE symptoms than patients who did not, though this was not a large difference (361 vs. 453). By way of contrast, IDS-atypical patients 
Figure 3: Number of profiles of depressive symptoms (i.e., not considering the specifier symptoms) for patients with MDD, with or without melancholic features, and with or without atypical features

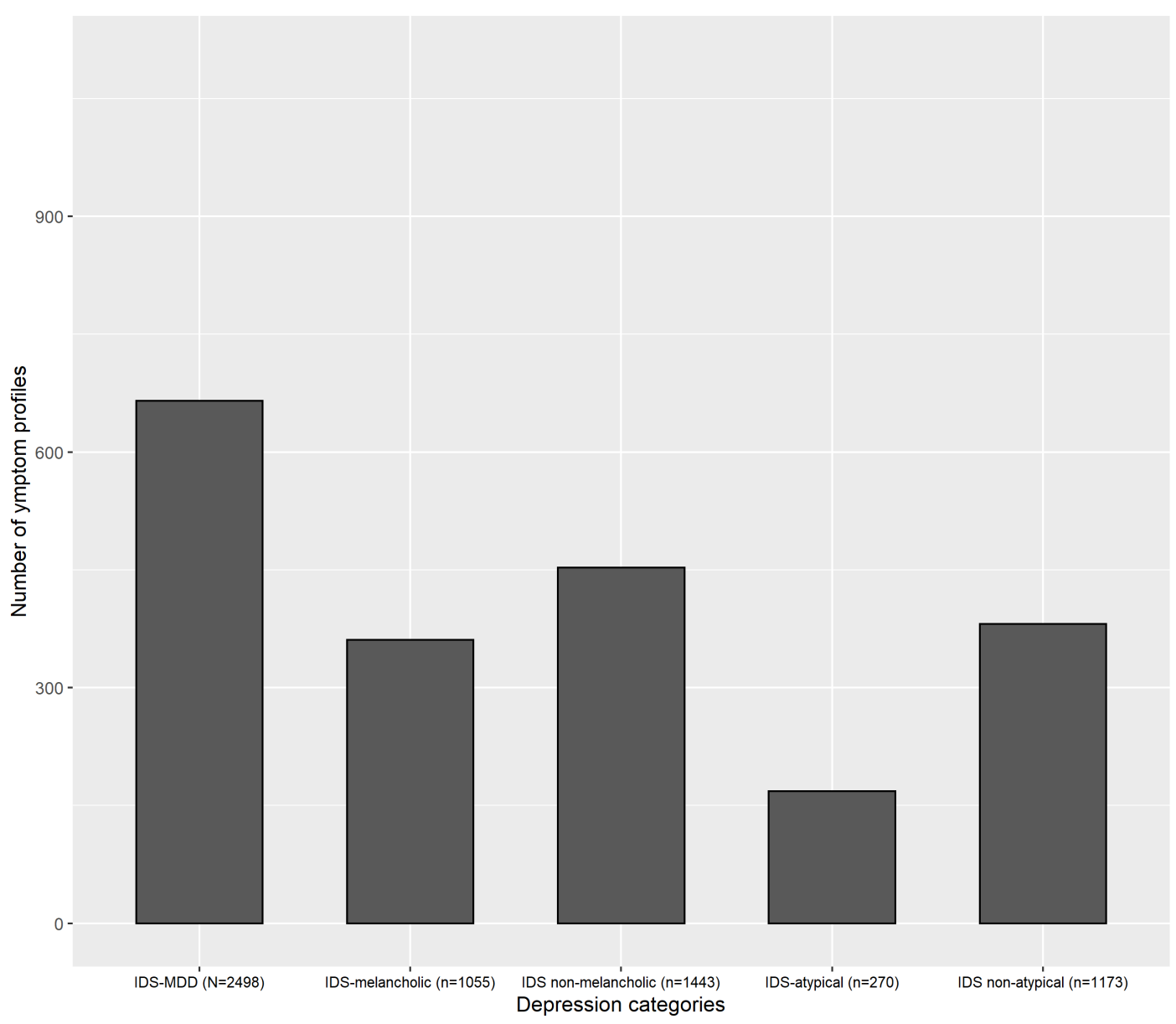

only had 168 combinations of MDE symptoms, compared to 381 combinations for patients categorized as IDS-non-atypical. Most of the symptom profiles for MDD ( $\mathrm{n}=569,85.56 \%$ ), IDS-melancholia $(\mathrm{n}=328,90.86 \%$, and IDS-atypical $(\mathrm{n}=163,97.02 \%)$ were endorsed by 5 or fewer patients.

\section{Sensitivity analyses}

To compare the frequency of symptom combinations across the groups while equating on sample size, we randomly resampled 100 patients in each subgroup 1,000 times and counted the mean number of symptom profiles per 100 patients. As seen in Figure 3, panel 
Figure 4: Number of unique symptom profiles of depression and its specifiers (Panel A) or depressive symptoms alone (Panel B) across 1,000 subsamples of $\mathrm{n}=100$ for IDS-MDD, IDS-melancholic, IDS nonmelancholic, IDS-atypical, and IDS non-atypical
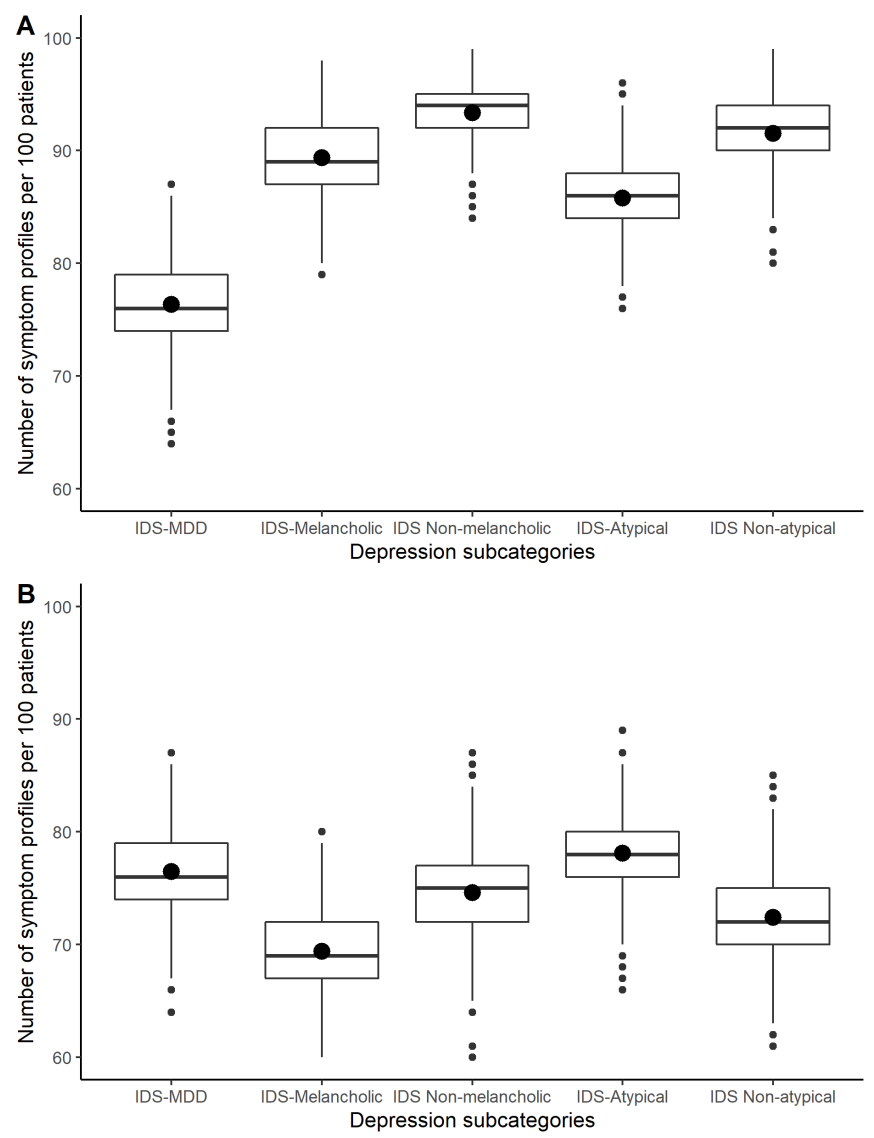

A, considering the depression symptoms as well as the specifier symptoms, there was more heterogeneity in the specifiers than in IDS-MDE alone. When focusing only on the symptoms of depression, there was some evidence that there were fewer profiles in the IDS-melancholic group $($ Mean $(\mathrm{M})=69.2$, Median $(\mathrm{Me})=69)$ than the IDS-MDE $(\mathrm{M}=76.47, \mathrm{Me}=76)$ and IDS-non-melancholic $(\mathrm{M}=74.62, \mathrm{Me}=75)$. Next, we re-ran these analyses we relaxing the DSM hierarchical rule that prohibits the diagnosis of atypical features if a person meets criteria for melancholic features. More individuals met IDS-atypical criteria if we relaxed this rule $(\mathrm{n}=348)$ but there was no evidence this was associated with lower heterogeneity (see Supplement). Additionally, we explored whether any specific symptom, as opposed to specifier groups, were associated with reduced heterogeneity. The results of these analyses suggest that the number of unique symptom combination is associated to the group size 
such that more infrequently-endorsed symptoms appear less heterogenenous by virtue of having fewer individuals in the subgroup but no symptom appeared to reduce heterogeneity considerably.

\section{Discussion}

We conducted what is, to our knowledge, the first exploration of whether the DSM specifiers for major depression achieve their intended purpose of creating more homogeneous patient subgroups. We computed both the number of unique "symptoms profiles" that result from the specifier criteria and the main symptom criteria for MDE as well as the number of unique symptoms profiles of the MDE symptoms alone. We failed to find consistent evidence that using the DSM specifiers for atypical and melancholic depression identifies a more homogeneous group of patients. The melancholic specifier appeared to reduce the number of overall combinations of the MDE symptoms, but there were more combinations of MDE and melancholic symptoms than there were unique ways of meeting MDE criteria alone. Across some analyses, patients with atypical depression appeared to have fewer symptom profiles than patients without atypical depression or with melancholic depression. However, we found that the atypical specifier, and the symptoms that appear to be associated with reduced symptom heterogeneity were simply rarer. Equating the number of patients present in the groups by subsampling revealed that the atypical specifiers applies to a group of patients that is just as heterogeneous or is more heterogeneous than the MDE criteria. Most of the profiles were endorsed by five or fewer patients. Thus, there did not appear to be consistent subgroups that were associated with reduced heterogeneity above and beyond a lower frequency of individuals meeting criteria for a given specifier.

\section{Limitations and strengths}

Patients were excluded from the $\mathrm{STAR}^{*} \mathrm{D}$ study if they reported psychotic symptoms or bipolar disorder as well as if they were deemed to have primary OCD, substance dependence, 
and prior non-response to the Level 1 medication. Thus, our results cannot be generalized to all patients undergoing a major depressive episode (e.g., those with a MDE in the context of bipolar II) nor all patients meeting criteria for a unipolar MDE. Moreover, we could only analyze symptoms related to the melancholic and atypical specifiers and did not explore psychotic, mixed, anxious, or other specifiers of depression.

Despite not being able to generalize our findings to all patients with a MDE, the STAR ${ }^{*} \mathrm{D}$ exclusion criteria are relatively commonplace in clinical trials(Lorenzo-Luaces et al., 2018). Moreover, the $\mathrm{STAR}^{*} \mathrm{D}$ sample is a rather large clinical sample. Patients were recruited with relatively minimal entry criteria from both primary and secondary care, increasing external validity. These data had indicators of all the symptoms contained in the DSM as well as the melancholic and atypical specifiers. Moreover, we made several decisions that likely downplayed the degree of heterogeneity added by specific symptoms. First, we treated all forms of insomnia (middle, late, and early) as the same symptom of depression. Similarly, we treated changes in appetite as the same as changes in weight, though this is another source of heterogeneity in the symptoms.

\section{Implications}

Our results provide little support for the idea that the DSM major depression specifiers reduce heterogeneity in symptom presentations, above and beyond simply identifying smaller groups of patients. Melancholia is often touted as a biological specifier of depression identifying a relatively homogeneous group of patients. Our results do not support this assertion, at least when it is measured by number of unique symptom profiles. Some have argued that the DSM definition of melancholia may not capture the "true" construct of melancholia, in part because it is bound by the DSM definition of depression (i.e., a patient cannot meet for the melancholic specifier without first meeting criteria for the problematic MDE criteria). If melancholia is to be a useful construct, a central task for research will be to define its necessary features (Lorenzo-Luaces et al., 2020). Parker and colleagues have identified psychomotor disturbances and disproportionate reactions to stressors as hallmarks of the 
melancholic affliction (Parker, 2005; Parker et al., 2013). Nonetheless, neither one of these are necessary for the DSM specifier, even as the manual notes that psychomotor disturbances are "nearly always present" (p. 151). (Incidentally, these data argue against the ubiquity of psychomotor disturbances in melancholic depression, see Table 1.) Psychomotor retardation may appear to reduce heterogeneity in symptom presentations but only because it is an infrequently endorsed symptom of depression (see Supplement). Accordingly, a rarer symptom (e.g., psychosis) would appear to be more effective in reducing heterogeneity. Moreover, the reduction of heterogeneity given by the use of rare symptoms should not be consider the best way to reduce variability. For example, even among patients with psychomotor retardation there were almost as many combinations of depressive, melancholic, and atypical as there were individuals who had psychomotor retardation.

At first pass, our results may have be taken to suggest that the atypical specifier reduced heterogeneity more than the melancholic specifier. While the atypical specifier precludes a patient from having melancholia, our secondary analyses relaxing this criterion did not change our pattern of results. Instead, it appeared to be the case that there were fewer profiles associated to atypical depression simply because there were fewer patients, possibly because the cardinal feature of atypical depression, mood reactivity $(42.47 \%)$, was less frequently endorsed than the loss of pleasure (60.37\%) or lack of mood reactivity $(57.53 \%)$ which characterizes melancholic features .

Various lines of evidence converge to undermine the validity of the DSM diagnostic specifiers. First, if one relaxes the DSM hierarchical rule of melancholia over atypical, the specifiers often co-occur suggesting that nothing about the DSM criteria identify unique groups (Arnow et al., 2015). Second, existing evidence suggests that the specifiers are not temporally stable (Melartin et al., 2004). Third, the specifiers do not appear to have prognostic or predictive value (Angst et al., 2007; Cuijpers et al., 2017), at least in predicting response to antidepressants or cognitive behavioral therapy. Finally, various contemporary approaches to the conceptualization of psychopathology undermine the DSM's categorization of mental 
disorders, including its categorization of specifiers. Chief among these are the NIMH RDoC criteria as well as the network approach to psychopathology which conceptualizes mental disorders as emerging from the dynamic interactions of symptoms and processes. Our results suggest that the specifiers as currently implemented do not decrease heterogeneity. Future proposals to decrease heterogeneity should be demonstrated empirically and not assumed. In particular, we recommend that researchers who explore heterogeneity between groups of patients consider the effect of subgroup sample size on apparently variability. 


\section{References}

American Psychiatric Association. (2013). Diagnostic and statistical manual of mental disorders (DSM-5). American Psychiatric Publications.

Angst, J., Gamma, A., Benazzi, F., Ajdacic, V., \& Rössler, W. (2007). Melancholia and atypical depression in the zurich study: Epidemiology, clinical characteristics, course, comorbidity and personality. Acta Psychiatrica Scandinavica, 115, 72-84.

Arnow, B. A., Blasey, C., Williams, L. M., Palmer, D. M., Rekshan, W., Schatzberg, A. F., Etkin, A., Kulkarni, J., Luther, J. F., \& Rush, A. J. (2015). Depression subtypes in predicting antidepressant response: A report from the iSPOT-D trial. American Journal of Psychiatry, 172(8), 743-750.

Association, A. P. Et al. (1980). Diagnostic and statistical mental disorder (tech. rep.). DSM-III. Washington: American Psychiatric Association.

Association, A. P. Et al. (2000). Diagnostic and statistical mental disorder (tech. rep.). DSM-IV-TR. Washington: American Psychiatric Association.

Baumeister, H., \& Parker, G. (2012). Meta-review of depressive subtyping models. Journal of Affective Disorders, 139(2), 126-140.

Bühler, J., Seemüller, F., \& Läge, D. (2014). The predictive power of subgroups: An empirical approach to identify depressive symptom patterns that predict response to treatment. Journal of Affective Disorders, 163, 81-87.

Cai, N., Choi, K. W., \& Fried, E. I. (2020). Reviewing the genetics of heterogeneity in depression: Operationalizations, manifestations, and etiologies. Human Molecular Genetics. Advanced online publication.

Cuijpers, P., Weitz, E., Lamers, F., Penninx, B. W., Twisk, J., DeRubeis, R. J., Dimidjian, S., Dunlop, B. W., Jarrett, R. B., Segal, Z. V., Et al. (2017). Melancholic and atypical depression as predictor and moderator of outcome in cognitive behavior therapy and pharmacotherapy for adult depression. Depression and Anxiety, 34(3), 246-256. 
Fava, M., Rush, A. J., Trivedi, M. H., Nierenberg, A. A., Thase, M. E., Sackeim, H. A., Quitkin, F. M., Wisniewski, S., Lavori, P. W., Rosenbaum, J. F., Et al. (2003). Background and rationale for the sequenced treatment alternatives to relieve depression (STAR* D) study. Psychiatric Clinics of North America.

Fried, E. I., Coomans, F., \& Lorenzo-Luaces, L. (2020). The 341737 ways of qualifying for the melancholic specifier. The Lancet Psychiatry, 7(6), 479-480.

Fried, E. I., \& Nesse, R. M. (2015a). Depression is not a consistent syndrome: An investigation of unique symptom patterns in the STAR* D study. Journal of Affective Disorders, 172, 96-102.

Fried, E. I., \& Nesse, R. M. (2015b). Depression sum-scores don't add up: Why analyzing specific depression symptoms is essential. BMC Medicine, 13(1), 72.

Horwitz, A. V., \& Wakefield, J. C. (2007). The loss of sadness: How psychiatry transformed normal sorrow into depressive disorder. Oxford University Press.

Horwitz, A. V., Wakefield, J. C., \& Lorenzo-Luaces, L. (2016). History of depression. The Oxford handbook of mood disorders, 11-23.

Juruena, M. F., Bocharova, M., Agustini, B., \& Young, A. H. (2018). Atypical depression and non-atypical depression: Is HPA axis function a biomarker? a systematic review. Journal of Affective Disorders, 233, 45-67.

Kessing, L. (2007). Epidemiology of subtypes of depression. Acta Psychiatrica Scandinavica, $115,85-89$.

Łojko, D., \& Rybakowski, J. K. (2017). Atypical depression: Current perspectives. Neuropsychiatric Disease and Treatment, 13, 2447.

Lorenzo-Luaces, L. (2015). Heterogeneity in the prognosis of major depression: From the common cold to a highly debilitating and recurrent illness. Epidemiology and Psychiatric Sciences, $24(6), 466-472$.

Lorenzo-Luaces, L., DeRubeis, R. J., van Straten, A., \& Tiemens, B. (2017). A prognostic index (pi) as a moderator of outcomes in the treatment of depression: A proof of 
concept combining multiple variables to inform risk-stratified stepped care models. Journal of Affective Disorders, 213, 78-85.

Lorenzo-Luaces, L., Rutter, L. A., \& Scalco, M. D. (2020). Carving depression at its joints? psychometric properties of the Sydney Melancholia Prototype Index. Psychiatry Research, 113410.

Lorenzo-Luaces, L., Zimmerman, M., \& Cuijpers, P. (2018). Are studies of psychotherapies for depression more or less generalizable than studies of antidepressants? Journal of Affective Disorders, 234, 8-13.

McGlinchey, J. B., Zimmerman, M., Young, D., \& Chelminski, I. (2006). Diagnosing major depressive disorder VIII: Are some symptoms better than others? The Journal of Nervous and Mental Disease, 194(10), 785-790.

Melartin, T., Leskelae, U., Rytsaelae, H., Sokero, P., LestelÄ-Mielonen, P., \& Isometsae, E. (2004). Co-morbidity and stability of melancholic features in DSM-IV major depressive disorder. Psychological Medicine, 34(8), 1443-1452.

Pae, C.-U., Tharwani, H., Marks, D. M., Masand, P. S., \& Patkar, A. A. (2009). Atypical depression. CNS Drugs, 23(12), 1023-1037.

Parker, G. (2005). Beyond major depression. Psychological Medicine, 35(4), 467-474.

Parker, G., McCraw, S., Blanch, B., Hadzi-Pavlovic, D., Synnott, H., \& Rees, A.-M. (2013). Discriminating melancholic and non-melancholic depression by prototypic clinical features. Journal of Affective Disorders, 144(3), 199-207.

Rush, A. J., Fava, M., Wisniewski, S. R., Lavori, P. W., Trivedi, M. H., Sackeim, H. A., Thase, M. E., Nierenberg, A. A., Quitkin, F. M., Kashner, T. M., Et al. (2004). Sequenced treatment alternatives to relieve depression STAR* D: Rationale and design. Controlled Clinical Trials, 25(1), 119-142.

Rush, A. J., Gullion, C. M., Basco, M. R., Jarrett, R. B., \& Trivedi, M. H. (1996). The inventory of depressive symptomatology (IDS): Psychometric properties. Psychological Medicine, 26(3), 477-486. 
Shorter, E. (2007). The doctrine of the two depressions in historical perspective. Acta Psychiatrica Scandinavica, 115, 5-13.

Stewart, J., McGrath, P., Quitkin, F., \& Klein, D. (2007). Atypical depression: Current status and relevance to melancholia. Acta Psychiatrica Scandinavica, 115, 58-71.

Trivedi, M. H., Rush, A. J., Wisniewski, S. R., Nierenberg, A. A., Warden, D., Ritz, L., Norquist, G., Howland, R. H., Lebowitz, B., McGrath, P. J., Et al. (2006). Evaluation of outcomes with citalopram for depression using measurement-based care in star* d: Implications for clinical practice. American journal of Psychiatry, 163(1), 28-40.

Türkçapar, M. H., Akdemir, A., Örsel, S. D., Demirergi, N., Sirin, A., Kiliç, E. Z., \& Özbay, M. H. (1999). The validity of diagnosis of melancholic depression according to different diagnostic systems. Journal of Affective Disorders, 54(1-2), 101-107.

Uher, R., Dernovsek, M. Z., Mors, O., Hauser, J., Souery, D., Zobel, A., Maier, W., Henigsberg, N., Kalember, P., Rietschel, M., Et al. (2011). Melancholic, atypical and anxious depression subtypes and outcome of treatment with escitalopram and nortriptyline. Journal of Affective Disorders, 132(1-2), 112-120.

Ulbricht, C. M., Dumenci, L., Rothschild, A. J., \& Lapane, K. L. (2018). Changes in depression subtypes among men in $\mathrm{STAR}^{*} \mathrm{D}$ : A latent transition analysis. American Journal of Men's Health, 12(1), 5-13.

Wakefield, J. C., \& Schmitz, M. F. (2013a). Can the DSM's major depression bereavement exclusion be validly extended to other stressors?: Evidence from the NCS. Acta Psychiatrica Scandinavica, 128(4), 294-305.

Wakefield, J. C., \& Schmitz, M. F. (2013b). Normal vs. disordered bereavement-related depression: Are the differences real or tautological? Acta Psychiatrica Scandinavica, $127(2), 159-168$.

Wakefield, J. C., \& Schmitz, M. F. (2013c). When does depression become a disorder? using recurrence rates to evaluate the validity of proposed changes in major depression diagnostic thresholds. World Psychiatry, 12(1), 44-52. 
Wakefield, J. C., Schmitz, M. F., First, M. B., \& Horwitz, A. V. (2007). Extending the bereavement exclusion for major depression to other losses: Evidence from the National Comorbidity Survey. Archives of General Psychiatry, 64(4), 433-440.

Zimmerman, M., Ellison, W., Young, D., Chelminski, I., \& Dalrymple, K. (2015). How many different ways do patients meet the diagnostic criteria for major depressive disorder? Comprehensive Psychiatry, 56, 29-34.

Zimmerman, M., \& Mattia, J. I. (2001). A self-report scale to help make psychiatric diagnoses: The psychiatric diagnostic screening questionnaire. Archives of General Psychiatry, 58(8), 787-794.

Zimmerman, M., McGlinchey, J. B., Chelminski, I., \& Young, D. (2006). Diagnosing major depressive disorder V: Applying the DSM-IV exclusion criteria in clinical practice. The Journal of Nervous and Mental Disease, 194(7), 530-533. 